Georgian Mathematical Journal

1(1994), No. 5, 477-484

\title{
ON SOME PROPERTIES OF SOLUTIONS OF SECOND ORDER LINEAR FUNCTIONAL DIFFERENTIAL EQUATIONS
}

\author{
I. KIGURADZE
}

\begin{abstract}
The properties of solutions of the equation $u^{\prime \prime}(t)=$ $p_{1}(t) u\left(\tau_{1}(t)\right)+p_{2}(t) u^{\prime}\left(\tau_{2}(t)\right)$ are investigated where $p_{i}:[a,+\infty[\rightarrow R$ $(i=1,2)$ are locally summable functions, $\tau_{1}:[a,+\infty[\rightarrow R$ is a measurable function and $\tau_{2}:[a,+\infty[\rightarrow R$ is a nondecreasing locally absolutely continuous one. Moreover, $\tau_{i}(t) \geq t(i=1,2), p_{1}(t) \geq 0$, $p_{2}^{2}(t) \leq(4-\varepsilon) \tau_{2}^{\prime}(t) p_{1}(t), \varepsilon=$ const $>0$ and $\int_{a}^{+\infty}\left(\tau_{1}(t)-t\right) p_{1}(t) d t<$ $+\infty$. In particular, it is proved that solutions whose derivatives are square integrable on $[a,+\infty$ [ form a one-dimensional linear space and for any such solution to vanish at infinity it is necessary and sufficient that $\int_{a}^{+\infty} t p_{1}(t) d t=+\infty$.
\end{abstract}

Consider the differential equation

$$
u^{\prime \prime}(t)=p_{1}(t) u\left(\tau_{1}(t)\right)+p_{2}(t) u^{\prime}\left(\tau_{2}(t)\right),
$$

where $p_{i}:\left[a,+\infty\left[\rightarrow R(i=1,2)\right.\right.$ are locally summable functions, $\tau_{i}$ : $[a,+\infty[\rightarrow R(i=1,2)$ are measurable functions and

$$
\tau_{i}(t) \geq t \quad \text { for } \quad t \geq a \quad(i=1,2) .
$$

We say that a solution $u$ of the equation (1) is a Kneser-type solution if it satisfies the inequality $u^{\prime}(t) u(t) \leq 0$ for $t \geq a_{0}$ for some $a_{0} \in[a,+\infty[$. A set of such solutions is denoted by $K$. By $W$ we denote a space of solutions of (1) that satisfy $\int_{a}^{+\infty} u^{\prime 2}(t) d t<+\infty$. The results of [1,2] imply that if $p_{1}(t) \geq 0$ for $t \geq a$ and the condition

$$
\text { (i) } \tau_{i}(t) \equiv t, \quad(i=1,2), \quad \int_{a}^{+\infty}\left|p_{2}(t)\right| d t<+\infty,
$$

1991 Mathematics Subject Classification. 34K15. 
or

(ii) $p_{2}(t) \leq 0$, for $t \geq 0, \quad \int_{a}^{+\infty} s p_{1}(s) d s<+\infty, \quad \int_{a}^{+\infty} \frac{s}{\tau_{2}(s)}\left|p_{2}(s)\right| d s<+\infty$,

holds, then $W \supset K$ and $K$ is a one-dimensional linear space. The case when the conditions (i) and (ii) are violated, the matter of dimension of $K$ and $W$ and their interconnection has actually remained unstudied. An attempt is made in this note to fill up this gap to a certain extent.

Theorem 1. Let $\tau_{i}(t) \geq t(i=1,2), p_{1}(t) \geq 0$ for $t \geq a$,

$$
\int_{a}^{+\infty}\left[\tau_{1}(t)-t\right] p_{1}(t) d t<+\infty
$$

and let $\tau_{2}$ be a nondecreasing locally absolutely continuous function satisfying

$$
p_{2}^{2}(t) \leq(4-\varepsilon) \tau_{2}^{\prime}(t) p_{1}(t) \text { for } t \geq a,
$$

where $\varepsilon=$ const $>0$. Then

$$
W \subset K, \quad \operatorname{dim} W=1 .
$$

Before proceeding to the proof of the theorem we shall give two auxiliary statements.

Lemma 1. Let the conditions of Theorem 1 be fulfilled and let $a_{0} \in$ $[a,+\infty[$ be large enough for the equality

$$
\int_{a_{0}}^{+\infty}\left[\tau_{1}(s)-s\right] p_{1}(s) d s \leq 4 \delta^{2}
$$

where $\delta=\frac{1}{4}\left[2-(4-\varepsilon)^{1 / 2}\right]$, to hold. Then any solution $u$ of the equation (1) satisfies

$$
\begin{aligned}
& \delta \int_{t}^{x}\left[u^{\prime 2}(s)+p_{1}(s) u^{2}(s)\right] d s \leq u^{\prime}(x) u(x)-u^{\prime}(t) u(t)+ \\
& \quad+(1-\delta) \int_{x}^{\tau(x)} u^{\prime 2}(s) d s \quad \text { for } \quad a_{0} \leq t \leq x<+\infty
\end{aligned}
$$




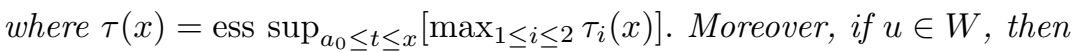

$$
u^{\prime}(t) u(t) \leq-\delta \int_{t}^{+\infty}\left[{u^{\prime}}^{2}(s)+p_{1}(s) u^{2}(s)\right] d s \text { for } t \geq a_{0}
$$

and

$$
2 \delta \int_{t}^{+\infty}(s-t)\left[u^{\prime 2}(s)+p_{1}(s) u^{2}(s)\right] d s \leq u^{2}(t) \text { for } t \geq a_{0} .
$$

Proof. Let $u$ be any solution of the equation (1). Then

$$
-u^{\prime \prime}(t) u(t)+p_{1}(t) u^{2}(t)=p_{1}(t) u(t) \int_{\tau_{1}(t)}^{t} u^{\prime}(s) d s-p_{2}(t) u^{\prime}\left(\tau_{2}(t)\right) u(t) .
$$

Integrating this equality from $t$ to $x$, we obtain

$$
\begin{aligned}
& u^{\prime}(t) u(t)-u^{\prime}(x) u(x)+\int_{t}^{x}\left[u^{\prime 2}(s)+p_{1}(s) u^{2}(s)\right] d s= \\
= & \int_{t}^{x}\left[p_{1}(s) u(s) \int_{\tau_{1}(s)}^{s} u^{\prime}(y) d y\right] d s-\int_{t}^{x} p_{2}(s) u^{\prime}\left(\tau_{2}(s)\right) u(s) d s .
\end{aligned}
$$

However, in view of (4) and (6),

$$
\begin{gathered}
\int_{t}^{x}\left[p_{1}(s) u(s) \int_{\tau_{1}(s)}^{s} u^{\prime}(y) d y\right] d s \leq \delta \int_{t}^{x} p_{1}(s) u^{2}(s) d s+ \\
+\frac{1}{4 \delta}\left[\int_{t}^{x}\left[\tau_{1}(s)-s\right] p_{1}(s) d s\right]\left[\int_{t}^{\tau(x)} u^{\prime 2}(s) d s\right] \leq \\
\leq \delta \int_{t}^{x} p_{1}(s) u^{2}(s) d s+\delta \int_{t}^{\tau(x)} u^{\prime 2}(s) d s \text { for } a_{0} \leq t \leq x<+\infty
\end{gathered}
$$

and

$$
-\int_{t}^{x} p_{2}(s) u^{\prime}\left(\tau_{2}(s)\right) u(s) d s \leq
$$




$$
\begin{gathered}
\leq 2(1-2 \delta) \int_{t}^{x}\left[p_{1}(s) u^{2}(s)\right]^{1 / 2}\left[\tau_{2}^{\prime}(s) u^{\prime 2}\left(\tau_{2}(s)\right)\right]^{1 / 2} d s \leq \\
\leq(1-2 \delta) \int_{t}^{x} p_{1}(s) u^{2}(s) d s+(1-2 \delta) \int_{t}^{x} \tau_{2}^{\prime}(s) u^{\prime 2}\left(\tau_{2}(s)\right) d s \leq \\
\leq(1-2 \delta) \int_{t}^{x} p_{1}(s) u^{2}(s) d s+(1-2 \delta) \int_{t}^{\tau(x)} u^{\prime 2}(s) d s \\
\text { for } a_{0} \leq t \leq x<+\infty .
\end{gathered}
$$

Therefore

$$
\begin{gathered}
u^{\prime}(t) u(t)-u^{\prime}(x) u(x)+\int_{t}^{x}\left[u^{\prime 2}(s)+p_{1}(s) u^{2}(s)\right] d s \leq \\
\leq(1-\delta) \int_{t}^{x}\left[{u^{\prime}}^{2}(s)+p_{1}(s) u^{2}(s)\right] d s+(1-\delta) \int_{x}^{\tau(x)} u^{\prime 2}(s) d s \\
\text { for } a_{0} \leq t \leq x<+\infty
\end{gathered}
$$

and thus the inequality (7) holds.

Suppose now that $u \in W$. Then, as one can easily verify,

$$
\liminf _{x \rightarrow+\infty}\left|u^{\prime}(x) u(x)\right|=0 .
$$

So (7) immediately implies (8). Integrating both sides of (8) from $t$ to $+\infty$, we obtain the estimate $(9)$.

Lemma 2. Let the conditions of Lemma 1 be fulfilled and there exist $b \in] a_{0},+\infty[$ such that

$$
p_{i}(t)=0 \text { for } t \geq b \quad(i=1,2) .
$$

Then for any $c \in R$ there exists a unique solution of the equation (1) satisfying

$$
u\left(a_{0}\right)=c, \quad u^{\prime}(t)=0 \quad \text { for } t \geq b .
$$

Proof. In view of (2) and (10), for any $\alpha \in R$ the equation (1) has a unique solution $v(\cdot ; \alpha)$ satisfying $v(t ; \alpha)=\alpha$ for $b \leq t<+\infty$. Moreover, $v(t ; \alpha)=\alpha v(t ; 1)$. On the other hand, by Lemma 1 the function $v(\cdot ; 1):\left[a_{0},+\infty\left[\rightarrow R\right.\right.$ is non increasing and $v\left(a_{0} ; 1\right) \geq 1$. Therefore the function $u(\cdot)=\frac{c}{v\left(a_{0} ; 1\right)} v\left(a_{0} ; \cdot\right)$ is a unique solution of $(1),(11)$. 
Proof of Theorem 1. First of all we shall prove that for any $c \in R$ the equation (1) has at least one solution satisfying

$$
u\left(a_{0}\right)=c, \quad \int_{a_{0}}^{+\infty} u^{\prime 2}(s) d s<+\infty .
$$

For any natural $k$ put

$$
p_{i k}(t)=\left\{\begin{array}{ll}
p_{i}(t) & \text { for } \quad a_{0} \leq t \leq a_{0}+k \\
0 & \text { for } \quad t>a_{0}+k
\end{array} \quad(i=1,2) .\right.
$$

According to Lemma 2, for any $k$ the equation $u^{\prime \prime}(t)=p_{1 k}(t) u\left(\tau_{1}(t)\right)+$ $p_{2 k}(t) u^{\prime}\left(\tau_{2}(t)\right)$ has a unique solution $u_{k}$ satisfying

$$
u_{k}\left(a_{0}\right)=c, \quad u_{k}^{\prime}(t)=0 \text { for } t \geq a+k .
$$

On the other hand, by Lemma 1

$$
\left|u_{k}(t)\right| \leq|c| \text { for } t \geq a_{0}, \quad 2 \delta \int_{a_{0}}^{+\infty}\left(s-a_{0}\right) u_{k}^{\prime 2}(s) d s \leq c^{2} .
$$

Taking (2) and (13)-(15) into account, it is easy to show that the sequences $\left(u_{k}\right)_{k=1}^{+\infty}$ and $\left(u_{k}^{\prime}\right)_{k=1}^{+\infty}$ are uniformly bounded and equicontinuous on each closed subinterval of $\left[a_{0},+\infty[\right.$. Therefore, by the Arzela-Ascoli lemma, we can choose a subsequence $\left(u_{k_{m}}\right)_{m=1}^{+\infty}$ out of $\left(u_{k}\right)_{k=1}^{+\infty}$, which is uniformly convergent alongside with $\left(u_{k_{m}}^{\prime}\right)_{m=1}^{+\infty}$ on each closed subinterval of $\left[a,+\infty\left[\right.\right.$. By (13)-(15) the function $u(t)=\lim _{m \rightarrow+\infty} u_{k_{m}}(t)$ for $t \geq a$ is a solution of the problem (1), (12).

We have thus proved that $\operatorname{dim} W \geq 1$. On the other hand, by Lemma 1 any solution $u \in W$ satisfies (8) and is therefore a Kneser-type solution. To complete the proof it remains only to show that $\operatorname{dim} W \leq 1$, i.e., that the problem (1), (12) has at most one solution for any $c \in R$. Let $u_{1}$ and $u_{2}$ be two artbitrary solutions of this problem and $u_{0}(t)=u_{2}(t)-u_{1}(t)$. Since $u_{0} \in W$ and $u_{0}\left(a_{0}\right)=0$, by Lemma 1

$$
2 \int_{a_{0}}^{+\infty}\left(s-a_{0}\right) u_{0}^{\prime 2}(s) d s=0 \text { and } u_{0}(t)=0 \text { for } t \geq a_{0},
$$

i.e., $u_{1}(t) \equiv u_{2}(t)$.

Remark 1. The condition (4) of Theorem 1 cannot be replaced by the condition

$$
p_{2}^{2}(t) \leq(4+\varepsilon) \tau_{2}^{\prime}(t) p_{1}(t) \text { for } t \geq a \text {. }
$$


Indeed, consider the equation

$$
u^{\prime \prime}(t)=\frac{1}{(4+\varepsilon) t^{2}} u(t)-\frac{1}{t} u^{\prime}(t)
$$

satisfying all conditions of Theorem 1 except (4), instead of which the condition (16) is fulfilled. On the other hand, the equation (17) has the solutions $u_{i}(t)=t^{\lambda_{i}}(i=1,2)$, where $\lambda_{i}=(-1)^{i}(4+\varepsilon)^{-\frac{1}{2}}(i=1,2)$. Clearly, $u_{i} \in W$ $(i=1,2)$. Therefore in our case instead of (5) we have $K \subset W, \operatorname{dim} W=2$.

Corollary 1. Let the conditions of Theorem 1 be fulfilled. Let, moreover,

$$
p_{2}(t) \leq 0 \text { for } t \geq a \text {. }
$$

Then

$$
K=W, \quad \operatorname{dim} K=1 .
$$

Proof. Let $u \in K$. Then by virtue of (18) and the non-negativity of $p_{1}$ there exists $t_{0} \in\left[a,+\infty\left[\right.\right.$ such that $u(t) u^{\prime}(t) \leq 0, u^{\prime \prime}(t) u(t) \geq 0$ for $t \geq t_{0}$. Hence

$$
\int_{t_{0}}^{+\infty}{u^{\prime 2}}^{2}(s) d s \leq\left|u\left(t_{0}\right) u^{\prime}\left(t_{0}\right)\right| .
$$

Therefore $u \in W$. Thus we have proved that $W \supset K$. This fact, together with (5), implies (19).

A solution $u$ of the equation (1) will be called vanishing at infinity if

$$
\lim _{t \rightarrow+\infty} u(t)=0 .
$$

Theorem 2. Let the conditions of Theorem 1 be fulfilled. Then for any solution $u \in W$ to vanish at infinity it is necessary and sufficient that

$$
\int_{a}^{+\infty} s p_{1}(s) d s=+\infty .
$$

Proof. Let $u \in W$. Then by Lemma $1 u^{2}(t) \geq \eta$ for $t \geq a_{0}$, where $\eta=$ $\lim _{t \rightarrow+\infty} u^{2}(t)$, and $\int_{a_{0}}^{+\infty}\left(s-a_{0}\right) p_{1}(s) u^{2}(s) d s \leq u^{2}\left(a_{0}\right) / 2 \delta$. Hence it follows that (21) implies $\eta=0$, i.e., $u$ is a vanishing solution at infnity.

To complete the proof it is enough to establish that if

$$
\int_{a}^{+\infty} s p_{1}(s) d s<+\infty,
$$


then any nontrivial solution $u \in W$ tends to a nonzero limit as $t \rightarrow+\infty$. Let us assume the contrary: the equation (1) has a nontrivial solution $u \in W$ vanishing at infinity. Then by Lemma 1

$$
u(t) u^{\prime}(t) \leq 0, \quad \rho(t) \leq \eta^{2} u^{2}(t) \quad \text { for } \quad t \geq a_{0}
$$

where

$$
\rho(t)=\int_{t}^{+\infty}(s-t)\left[u^{\prime 2}(s)+p_{1}(s) u^{2}(s)\right] d s, \quad \eta=(2 \delta)^{-\frac{1}{2}} .
$$

On the other hand, by (4), (20) and (22) we have

$$
\begin{aligned}
& |u(t)|=\left|\int_{t}^{+\infty}(s-t)\left[p_{1}(s) u\left(\tau_{1}(s)\right)+p_{2}(s) u^{\prime}\left(\tau_{2}(s)\right)\right] d s\right| \leq \\
& \leq\left[\int_{t}^{+\infty}(s-t) p_{1}(s) d s\right]^{1 / 2}\left[\int_{t}^{+\infty}(s-t) p_{1}(s) u^{2}\left(\tau_{1}(s)\right) d s\right]^{1 / 2}+ \\
& \leq\left[\int_{t}^{+\infty}(s-t) p_{1}(s)\right]^{1 / 2}\left[\int_{t}^{+\infty}(s-t)\left[p_{1}(s)\right]^{1 / 2}\left[\tau_{2}^{\prime}(s)\right]^{1 / 2} \mid u^{\prime}\left(\tau_{2}(s) \mid d s \leq\right.\right. \\
& +2\left[\int_{t}^{+\infty}(s-t) p_{1}(s) d s\right]^{1 / 2}\left[\int_{t}^{+\infty}(s-t) \tau_{2}^{\prime}(s) u^{\prime 2}\left(\tau_{1}(s)\right) d s\right]^{1 / 2}+ \\
& +2 \text { for } t \geq a_{0} .
\end{aligned}
$$

Hence by (2) and (23) we find

$$
\begin{aligned}
&|u(t)| \leq {\left[\left.\int_{t}^{+\infty}(s-t) p_{1}(s) d s\right|^{1 / 2}\left[\int_{t}^{+\infty}(s-t) p_{1}(s) u^{2}(s) d s\right]^{1 / 2}+\right.} \\
&+2\left[\int_{t}^{+\infty}(s-t) p_{1}(s) d s\right]^{1 / 2}\left[\int_{t}^{+\infty}(s-t) u^{\prime 2}(s) d s\right]^{1 / 2} \leq \\
& \leq 3 \eta\left[\int_{t}^{+\infty}(s-t) p_{1}(s) d s\right]^{1 / 2}|u(t)| \text { for } t \geq a_{0}
\end{aligned}
$$


and therefore $u(t)=0$ for $t \geq a_{1}$, where $a_{1}$ is a sufficiently large number. By virtue of (2) the last equality implies $u(t)=0$ for $t \geq a$. But this is impossible, since by our assumption $u$ is a nontrivial solution. The obtained contradiction proves the theorem.

\section{REFERENCES}

1. I.T. Kiguradze, On the non-negative nonincreasing solutions of nonlinear second order differential equations. Ann. mat. pura ed appl. 81(1969), 169-192.

2. I.T. Kiguradze and D.I. Chichua, Kneser's problem for functional differential equations. (Russian) Diffferentsial'nye Uravneniya 27(1992), No. 11, 1879-1892.

(Received 03.08.1993)

Author's address:

A. Razmadze Mathematical Institute

Georgian Academy of Sciences

1, Z. Rukhadze St., Tbilisi 380093

Republic of Georgia 